\title{
Impacts of the side ratio of isolated buildings on airflows and pollutant dispersion under different temperature stratifications: Numerical investigations
}

\author{
Xiaohui Huang, Lizhen Gao ${ }^{\dagger}$ \\ College of Environmental Science and Engineering, Taiyuan University of Technology, Taiyuan, China
}

\begin{abstract}
Based on the Reynolds-averaged Navier-Stokes (RANS) method, the standard $k-\varepsilon$ turbulence model was used to simulate the effects of the side ratio (SR: building depth/building width, $S R=0.5,1.0,1.5$, and 2.0, respectively) of isolated buildings on the flow field and pollutant of ethylene dispersion under unstable temperature stratification and neutral conditions. The results showed that under unstable temperature stratification, the updraft on the leeward side of the building caused the high temperature gas to flow upward and the temperature gradient to drop. Compared with the neutral condition, the length of the recirculation zone was shorter, and with the decrease in SR, the vortex in the recirculation zone became larger and more stable. Because the pollution source was located on the leeward side of the building, the pollutant gas was concentrated in the area between the pollution source and the leeward side of the building. The near-ground concentration of pollutants under unstable temperature stratification was lower than that under neutral conditions, and with the decrease in $S R$, the near-ground axis concentration decreased while the width of the pollutant plumes increased.
\end{abstract}

Keywords: Isolated building, Numerical simulation, Pollutant dispersion, Side ratio, Temperature stratification

\section{Introduction}

The influence of buildings on nearby low emission sources was associated with many factors, such as building geometry, orientation, spacing, local meteorological conditions and complex urban structures. Common methods for studying the effects of buildings on the surrounding airflow and the pollutant distribution included field measurements, wind tunnel experiments and numerical simulations. Various single factors were studied by researchers. For example, the influence of uniform and nonuniform building height [1-6] and building layout [3, 7-9], building spacing with an aspect ratio of a street canyon [10-13], building shapes [14, 15], and fixed or time-varying meteorological conditions [8, 16-18] were investigated.

However, current wind tunnel tests and numerical simulations were mostly based on neutral conditions, while the actual atmosphere was often nonneutral [19]. A nonneutral boundary layer was a common atmospheric phenomenon. For example, unstable conditions usually occurred during a day due to human activities and the absorption of solar radiation by the ground. In contrast, under clear sky conditions at night, stable conditions appeared. Unlike under neutral conditions, the buoyancy effect caused by a temperature difference played a crucial role in the nonisothermal boundary layer. There was an adiabatic potential temperature profile in a neutral atmosphere, in which the vertical motion of fluid particles was neither amplified nor weakened. The vertical motion was weakened under stable conditions, whereas it was enhanced under unstable (or convective) conditions, while the vertical motion was an important determinant of pollutant diffusion [20]. Therefore, the influence of the thermal effect on air flow and pollutant dispersion had attracted extensive attention. Many researchers confirmed that atmospheric stability conditions had a strong influence on the dispersion of pollutants $[8,9,11$, 21-27]. Isolated buildings were the most basic units of complex urban structures. The study of flow and pollutant dispersion around isolated buildings was the basis of many urban area studies and it was suitable for a parametric study. Tominaga and Stathopoulos (2009) compared four types of turbulence models
This is an Open Access article distributed under the terms of the Creative Commons Attribution Non-Commercial License (http://creativecommons.org/licenses/by-nc/3.0/) which permits unrestricted non-commercial use, distribution, and reproduction in any medium, provided the original work is properly cited.

Copyright (C) 2022 Korean Society of Environmental Engineers
Received March 02, 2021 Accepted August 08, 2021

${ }^{\dagger}$ Corresponding author

E-mail: lzgao1@yahoo.com

Tel: +15803516556

ORCID: 0000-0001-7558-3042 
(the standard $k-\varepsilon$ model, the RNG $k-\varepsilon$ model, the $k-\varepsilon$ model with Launder and Kato modification and the realizable $k-\varepsilon$ model) in predicting the flow and dispersion around a cubic building with a flush vent located on its roof under neutral conditions [28]. It was proven that the standard $k-\varepsilon$ model provided inaccurate results for the concentration field due to the failure to reproduce the backflow of the building roof. However, the RNG $k-\varepsilon$ model was in good agreement with the experimental results. It could be inferred that accurately reproducing the flow, especially the flow field around a pollution source, was the key to accurately predicting a concentration field. Subsequently, Tominaga and Stathopoulos [29] compared the accuracy of the large eddy simulation (LES) and the RNG $k-\varepsilon$ model for the same building and source locations under neutral conditions. They concluded that LES performed better than RNG, but the computation time was approximately 25 times longer than in the RNG case. Similarly, they compared and investigated the effect of large-scale fluctuations on the velocity and concentration statistics [30, 31]. Gousseau et al. [32] conducted simulations of pollutant dispersion around isolated buildings to compare the RANS equations (the standard $k-\varepsilon$ model, the RNG $k-\varepsilon$ model, the realizable $k-\varepsilon$ model and the Reynolds-stress model (RSM) with a linear pressure-strain model and wall-reflection effects) and LES about the role of convective and turbulent mass fluxes under neutral conditions. Du et al. [33] proposed a novel model, transport-based recurrence CFD (rCFD), to overcome the drawback of the long computation time in LES. There were also studies of isolated buildings under different temperature stratification conditions. Mavroidis et al. [34-36] researched the residence time (the time required for a gas concentration to decay to $1 / e$ of its original value) of pollutants around an isolated cubical building under different atmospheric stability conditions through field experiments and numerical simulations. They found that the residence time was dependent on the atmospheric stability. By wind tunnel experiments, Yassin [23, 37] investigated the effects of thermal stability on the dispersion of roof stack emissions around a cubical building and found that the concentration in the near-wake zone increased under stable conditions and decreased under unstable conditions. So far, most previous studies focused on (i) the building layout, especially the building height and windward width, which were considered to have a more significant influence on the flow field; or (ii) selection of the turbulence model, RANS or LES; and (iii) the influence of temperature stratification.

In a numerical simulation, the LES method was more accurate than the RANS method [38, 39], mainly because the LES method could accurately reproduce unsteady periodic fluctuations around the building [40]. However, compared with neutral conditions, the generation of inlet conditions for LES was more difficult in nonisothermal conditions, as the turbulence intensity usually varied with different thermal stabilities. The application of LES in nonisothermal boundary layers was limited, as both inflow velocity fluctuations and temperature fluctuations were necessary for the simulation. In fact, although LES was more accurate, RANS modeling was still widely used due to the relatively lower computational cost and time required [41]. LES was generally recognized as a research tool rather than as an instrument to solve practical cases.

This study was proposed mainly from the considerations: (i) the coupling effect of temperature stratification and building $S R$ was rarely investigated, as mentioned by Zhang et al. [42]; (ii) taking an isolated building as a research object still had practical significance particularly for the environment of chemical plants. For environmental and safety concerns, some chemical plants with few buildings in close proximity could be considered as isolated buildings. In addition, ethylene was the most widely used chemical in the petrochemical industry. The study of the side ratio and temperature stratification had certain reference significance to optimize the design of chemical plants; (iii) the simulation results of the various turbulence models for the dispersion of pollution sources at different locations were different. Therefore, when a study focused on pollution dispersion, the turbulence model should be selected more prudently.

In this paper, based on the RANS method, the standard $k-\varepsilon$ turbulence model was used to simulate the effects of the $S R$ of buildings on the flow field and pollutant (ethylene) dispersion under neutral and unstable temperature stratification conditions. This paper was structured as follows: the validity of the model was described in Section 2, the simulation setup was introduced in Section 3, the main research results were introduced in Section 4, and the main conclusions were presented in Section 5.

\section{Model Validation}

\subsection{Outline of Wind Tunnel Experiments}

This study used the wind tunnel experiment results of Architectural Institute of Japan (AIJ) for model validation. The flow, temperature and concentration fields around a building within the non-isothermal boundary layer were selected for this database. The experiment was conducted in the thermally stratified wind tunnel (cross section at measurement part: $1.2 \mathrm{~m} \times 1.0 \mathrm{~m}$ ) of Tokyo Polytechnic University (TPU). The model building had a height $(H)$ of 160 $\mathrm{mm}$, a width $(W)$ of $80 \mathrm{~mm}$, and a depth $(D)$ of $80 \mathrm{~mm}(H: W: D$ $=2: 1: 1$, and was located in a turbulent boundary layer. The temperature conditions of the flow field were set as an unstable non-isothermal state $\left(R_{b}=-0.08\right)$.

Fig. 1 showed the vertical distributions of the approaching normalized wind velocity $u / U_{H}$, normalized temperature $\left(T-T_{f}\right) / \Delta T$, and normalized turbulent kinetic energy $k / U_{H}^{2}$. The Reynolds number based on $H$ (building height) and $U_{H}\left(U_{H}=1.46 \mathrm{~m} / \mathrm{s}\right.$, approaching wind velocity at building height) was about 15,000 . The wind tunnel floor temperature was $318.8 \mathrm{~K}$. A point of gas source was set on the floor $40 \mathrm{~mm}$ far from leeward of the model building. Tracer gas $\left(\mathrm{C}_{2} \mathrm{H}_{4}: 5 \%\right.$ ethylene) was released from a hole (diameter: $5 \mathrm{~mm}$ ) at a flow rate of $Q_{V}=9.17 \times 10^{-6} \mathrm{~m}^{3} / \mathrm{s}$ and the exit velocity was $0.47 \mathrm{~m} / \mathrm{s}$. Simultaneously, the wind velocity was measured by using a split film probe, air temperature was tested by a cold-wire technique, and gas concentration was analyzed by a fast response flame ionization detector. The sampling frequency was set at 1,000 $\mathrm{Hz}$ to obtain 90,000 data in $90 \mathrm{~s}$. The detailed techniques for simultaneously measuring fluctuating velocity, temperature and concentration in non-isothermal flow condition were similar as those described in the literature [43]. 

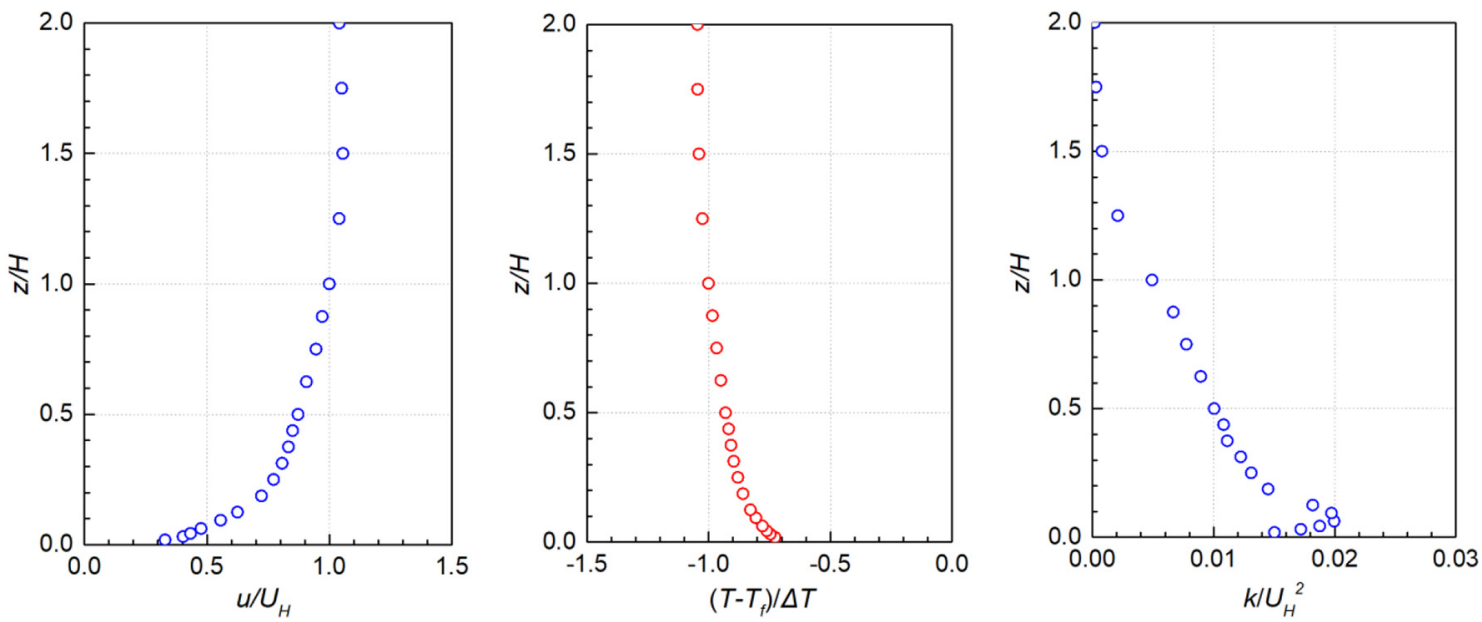

Fig. 1. The vertical distributions of the approaching flow for wind tunnel experiment.

\subsection{Simulation Settings and Verification}

\subsubsection{Computational domain and grid setup}

The size of the model building and pollution source in the simulation was consistent with the wind tunnel test. The calculation domain was set to $17 H \times 7.5 H \times 6.25 H$ (length $\times$ width $\times$ height, the width and height of the domain were consistent with those of the measured cross-sections in wind tunnel test). The blockage ratio was $1.0 \%$ which met the requirement of blockage ratio less than $3 \%$ for numerical simulation [44]. The basic grid adopted structured grid, building being discretized to $16(x) \times 16(y) \times 32(z)$. The grid height of the first layer adjacent to the ground surface was $0.008 \mathrm{~m}$; $y+$ was 22; the total number of the grids was 1.4 million.

\subsubsection{Numerical simulation method and boundary conditions}

The selection of the turbulence model strongly affected the prediction accuracy of the flow and dispersion around the building. As pointed out previously, Tominaga and Stathopoulos (2009) compared four types of $k-\varepsilon$ models for predicting the flow and dispersion around a cubic building with a flush vent located on its roof under neutral conditions and proved that the standard $k-\varepsilon$ model provided inaccurate results due to an inability to reproduce the backflow of the building roof, while the RNG $k-\varepsilon$ model was in good agreement with the experimental results. Although the object was an isolated building, the source of pollution in this study was located on the leeward side of the building and under unstable temperature stratification condition. Therefore, the standard $k-\varepsilon$ turbulence model (hereafter, SKE), RNG $k-\varepsilon$ turbulence model (hereafter, RNG) and realizable $k-\varepsilon$ turbulence model (hereafter, RLZ) were compared in this study.

The inflow wind velocity $u$, turbulent kinetic energy $k$ and temperature $T$ were also consistent with those set in wind tunnel test experiment. The value of $\varepsilon$ was obtained from the following equation, assuming local equilibrium of the production term $P_{k}$ in the $k$ equation: $\varepsilon \cong P_{k} \cong-u^{\prime} w^{\prime} \frac{\partial u}{\partial z}$, where $\left\langle u^{\prime} w^{\prime}\right\rangle$ was measured by wind tunnel experiment. The turbulent Schmidt number $\left(S C_{t}\right)$ was set at 0.7. The buildings walls and the ground were set as no-slip wall boundary conditions and standard wall functions were applied. Symmetric boundary conditions were applied to the two sides of the domain (parallel to the $x$-axis) and the top surface.

In the calculation, the Semi-Implicit Method for Pressure Linked Equations-Consistent (SIMPLEC) was used to couple the pressure and momentum equation, and the advection scheme was second-order Upwind Difference Scheme (UDS). In order to ensure the convergence of the calculation results, the residuals of all variables should be less than $10^{-4}$.

\subsubsection{Grid-independent study}

Except for the basic grid, the calculations were performed for a finer grid with $1 / 2$ of the basic grid's resolution. The basic conditions used were the same as for the other calculation conditions. Fig. 2 showed the different grid settings and the distributions of normalized wind velocity, temperature and concentration in the vertical section $(y / H=0)$ and near-ground section $(z / H=0.025)$ of the building. In Fig. 2, the vertical circle dotted lines exhibited the positions of each measurement line $(x / H=-0.25,0.25,0.625$, 1, 1.5 and 2) in the wind tunnel experiment. The normalized wind velocity, temperature and concentration values were plotted with these lines as the vertical axes. According to Fig. 2, the results for the basic and fine grids were almost identical, which indicated that the discretization of the basic grid was sufficient. Therefore, the basic grid was selected in this study.

\subsubsection{Simulation validation results}

According to Fig. 2, although CFD overestimated the length of the recirculation zone behind the building, the calculated vertical velocity distribution, temperature distribution and concentration distribution were almost consistent with the results of the wind tunnel tests, indicating that CFD could simulate unstable temperature stratifications. The discrepancies between the calculated and wind tunnel test values mainly appeared in the regions near the ground. This meant that it was still impossible for the CFD technique to accurately predict the near wall flow even with the LES model $[40,45]$. Comparing the different turbulence models, although the SKE model did not reproduce the negative value near the roof surface because of the reverse flow, the simulation of the velocity 

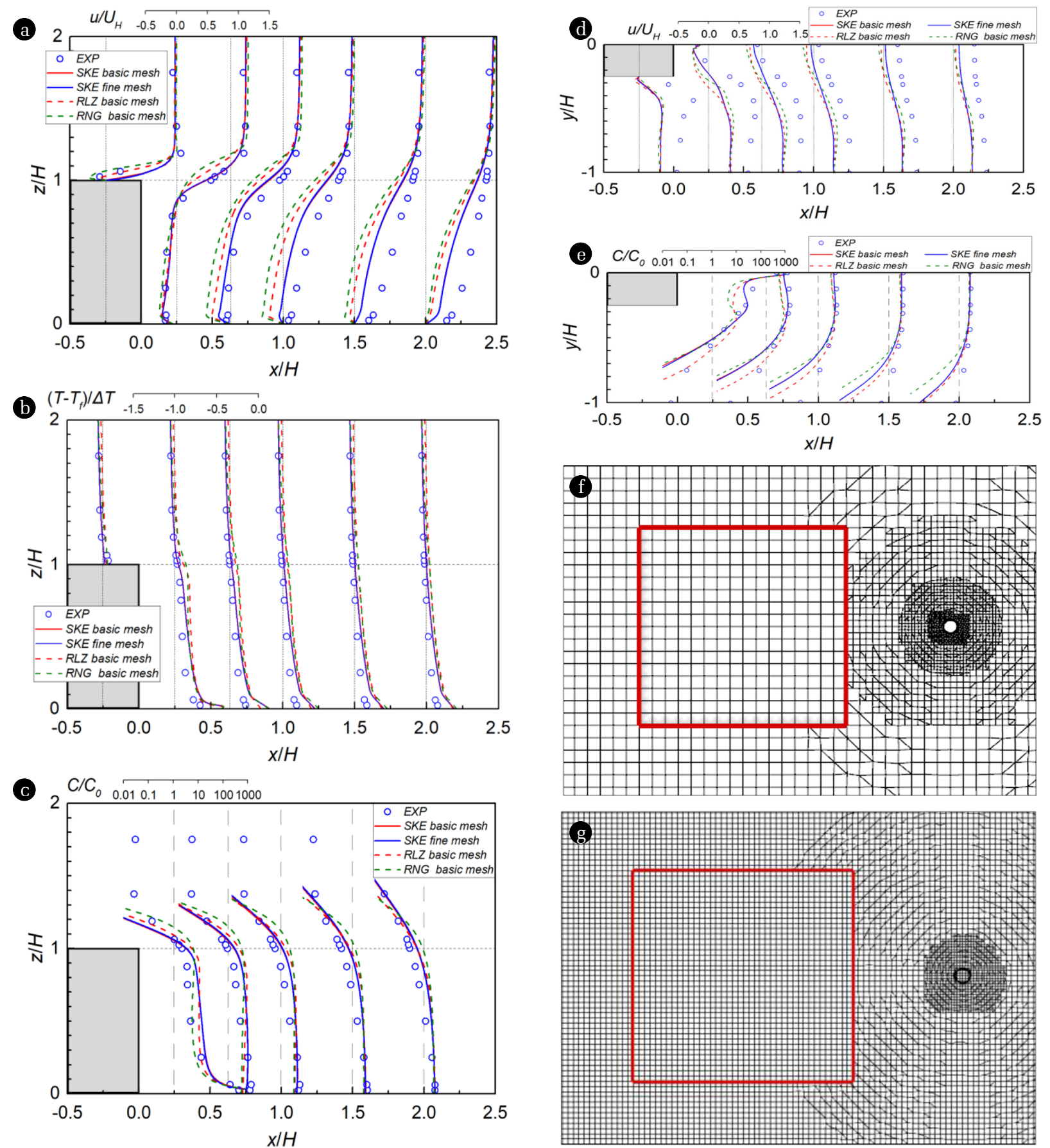

Fig. 2. (a), (b), (c) The distributions of normalized wind velocity, temperature and concentration in the vertical section $(y / H=0)(d)$, (e) and near-ground section $(\mathrm{z} / \mathrm{H}=0.025)(\mathrm{f})$ and the different grid settings: basic grid (g) and fine grid.

and temperature behind the building generally corresponded well with the experimental counterparts. This indicated that although the RNG model performed better than SKE in predicting the dispersion around buildings with roof emissions, this was not the case in this study. The reason for this was that the pollution source in this study was located downstream of the leeward side of the building, and the accuracy of the prediction of the flow field and velocity field on the leeward side was more critical. The AIJ also indicated that compared with the revised $k-\varepsilon$ models, the SKE model showed the best agreement with the experiment in weak wind regions, such as the recirculation zones [46]. Therefore, the SKE model was adopted in this study. 


\section{Simulation Setup}

\subsection{Model Case Settings and Main Parameters}

In this study, four kinds of building configurations were set and two kinds of temperature stratification (neutral and unstable temperature stratification) were studied. The building configurations, grid setting and inflow profile were presented in Fig. 3. The case settings and main parameters were listed in Table 1. In all cases, the building width $B$ was $80 \mathrm{~mm}$, the height $H$ was $160 \mathrm{~mm}$, and the depth $D$ was $160 \mathrm{~mm}, 120 \mathrm{~mm}, 80 \mathrm{~mm}$ and $40 \mathrm{~mm}$ respectively. The origin of coordinates was located in the center of the ground on the leeward side of the building. The source of pollution (ground point source) was located on the leeward of the building and the coordinates were $(0.25 \mathrm{H}, 0 \mathrm{H}, 0 \mathrm{H})$. Tracer gas $\left(\mathrm{C}_{2} \mathrm{H}_{4}: 5 \%\right.$ ethylene) was released from the source (hole diameter: $5 \mathrm{~mm}$ ) at a flow rate of $Q_{V}=9.17 \times 10^{-6} \mathrm{~m}^{3} / \mathrm{s}$ and the exit velocity was $0.47 \mathrm{~m} / \mathrm{s}$.

As the density of ethylene was very close to that of air and it was of negligible buoyancy effect, it was the most commonly used passive transport gas tracer. On the other hand, ethylene
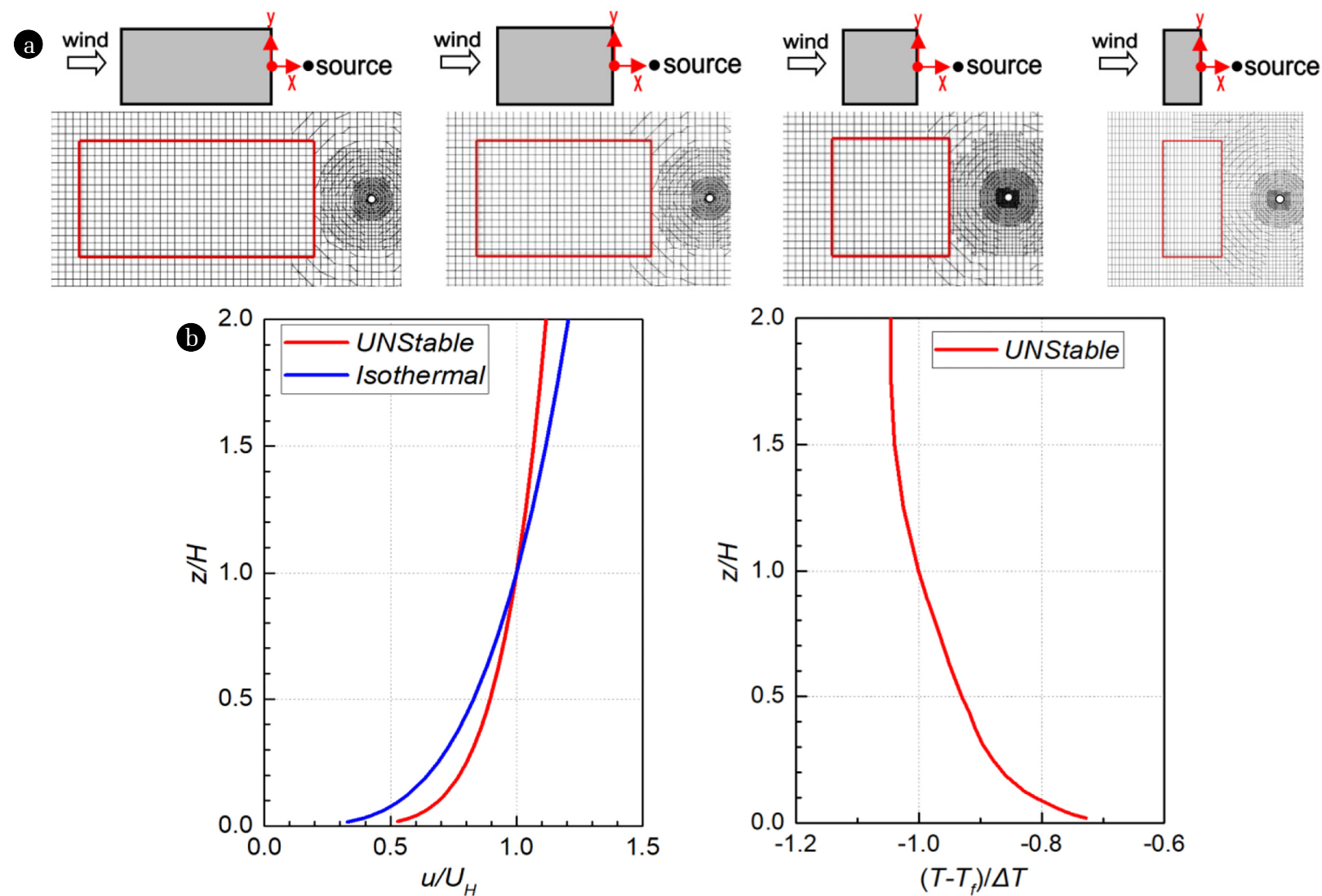

Fig. 3. (a) Building configurations (top view) (b) and the profile of vertical distribution of inflow.

Table 1. Computational Case Settings and Main Parameters

\begin{tabular}{|c|c|c|c|c|c|c|c|c|c|}
\hline Case No. & $\begin{array}{c}\text { Building size }(\mathrm{mm}) H=160 \mathrm{~mm} \\
(D \times B)\end{array}$ & $\begin{array}{l}\text { Temperature } \\
\text { stratifications }\end{array}$ & $\begin{array}{c}U_{H} \\
(\mathrm{~m} / \mathrm{s})\end{array}$ & $\begin{array}{c}T_{H} \\
(\mathrm{~K})\end{array}$ & $\begin{array}{c}T_{f} \\
(\mathbf{K})\end{array}$ & $\begin{array}{l}\Delta T \\
(\mathrm{~K})\end{array}$ & $\begin{array}{l}T_{g a s} \\
(\mathbf{K})\end{array}$ & $\begin{array}{c}\mathbf{u}^{*} \\
(\mathrm{~m} / \mathrm{s})\end{array}$ & $\begin{array}{l}z_{0} \\
(\mathrm{~m})\end{array}$ \\
\hline $\mathrm{CASE}[S R=2, \mathrm{~N}]$ & $160 \times 80(S R=2)$ & \multirow{4}{*}{$\begin{array}{l}\text { Neutral } \\
R_{b}=0\end{array}$} & \multirow{4}{*}{1.46} & \multirow{4}{*}{294.0} & \multirow{4}{*}{294.0} & \multirow{4}{*}{0} & \multirow{4}{*}{294.0} & \multirow{4}{*}{0.069} & \multirow{4}{*}{0.0003} \\
\hline $\mathrm{CASE}[S R=1.5, \mathrm{~N}]$ & $120 \times 80(S R=1.5)$ & & & & & & & & \\
\hline $\mathrm{CASE}[S R=1, \mathrm{~N}]$ & $80 \times 80(S R=1)$ & & & & & & & & \\
\hline $\mathrm{CASE}[S R=0.5, \mathrm{~N}]$ & $40 \times 80(S R=0.5)$ & & & & & & & & \\
\hline $\mathrm{CASE}[S R=2, \mathrm{UNS}]$ & $160 \times 80(S R=2)$ & \multirow{4}{*}{$\begin{array}{c}\text { Unstable } \\
R_{b}=-0.08\end{array}$} & \multirow{4}{*}{1.46} & \multirow{4}{*}{285.2} & \multirow{4}{*}{318.8} & \multirow{4}{*}{33.6} & \multirow{4}{*}{303.4} & \multirow{4}{*}{0.098} & \multirow{4}{*}{0.000} \\
\hline $\mathrm{CASE}[S R=1.5, \mathrm{UNS}]$ & $120 \times 80(S R=1.5)$ & & & & & & & & \\
\hline $\mathrm{CASE}[S R=1, \mathrm{UNS}]$ & $80 \times 80(S R=1)$ & & & & & & & & \\
\hline $\mathrm{CASE}[S R=0.5, \mathrm{UNS}]$ & $40 \times 80(S R=0.5)$ & & & & & & & & \\
\hline
\end{tabular}


was the most popularly used chemical in petrochemical industry. Here in this study ethylene was selected as the pollutant gas tracer as well.

\subsection{CFD Calculation Conditions}

The calculation domain was set to $17 H \times 7.5 H \times 6.25 H$ (length $\times$ width $\times$ height). The blockage ratio was $1.0 \%$. In the simulation, all cases were constructed with the basic grid. The numerical simulation methods and boundary conditions were consistent with the verification case, as shown in Section 2.2.2.


\section{Results and Discussion}

\subsection{Velocity Field and Structure of Flow Fields}

Fig. 4(a) and 4(b) showed the normalized velocity distribution of the near-ground $(z / H=0.025)$ and vertical profiles $(y / H=0)$. Temperature stratification and $S R$ had a certain degree of influence on the flow, especially for the lateral wind direction and the downwind direction of the building. Under neutral conditions, when $S R=2.0,1.5,1.0$, and 0.5 , the length of the recirculation zones was $1.0 \mathrm{H}, 1.2 \mathrm{H}, 1.5 \mathrm{H}$, and $1.9 \mathrm{H}$, respectively. It was found
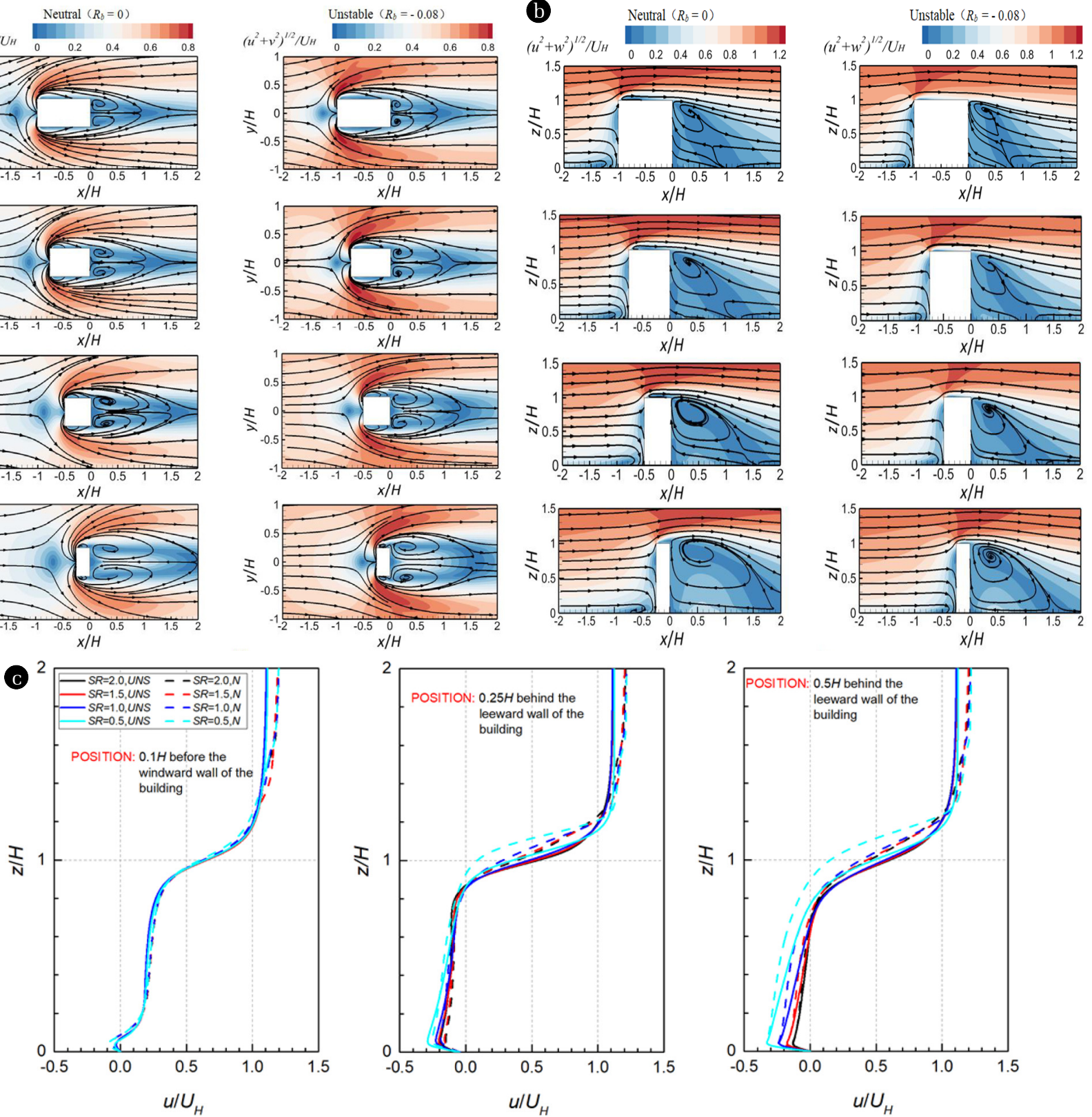

Fig. 4. Normalized velocity distribution (a) of near-surface $(z / H=0.025)$, (b) of vertical section $(y / H=0)$, (c) of fixing points before $(0.1 H$ before the windward side) and behind the building $(0.25 \mathrm{H}$ and $0.5 \mathrm{H}$ behind the leeward side). 
that the range of the recirculation zones increased and the shape of vortices became increasingly stable with decreasing SR. Moreover, with the decrease in $S R$, the lateral wind speed near the ground of the building was strengthened, and the transverse width of the leeward backflow area was increased. This was because the backflow wind speed increased with decreasing $S R$. In addition, when the building was longer (such as $S R \geq 1$ ), the airflow reattached at the side, making the airflow tighten somewhat, which was consistent with the results of Jiang and Yoshie [40]. Under unstable conditions, when $S R=2.0,1.5,1.0$, and 0.5 , the length of the recirculation zones was $0.8 H, 0.9 H, 1.1 H$, and $1.5 H$, respectively. This was the same trend as under neutral conditions. It was worth noting that, when the $S R$ was fixed, the length of the recirculation zones under the unstable condition was smaller than that under the neutral condition, and the shape of the vortex was more stable. This was because the vertical movement of the atmosphere was more intense and the reverse flow was also stronger under unstable conditions.

Fig. 4(c) compared the vertical distribution of $u / U_{H}$ at different positions in the vertical section $(y / H=0)$. As could be seen from Fig. 4(c), at $0.1 H$ before the windward side of the building, the $S R$ and the temperature stratification had little influence on $u / U_{H}$.
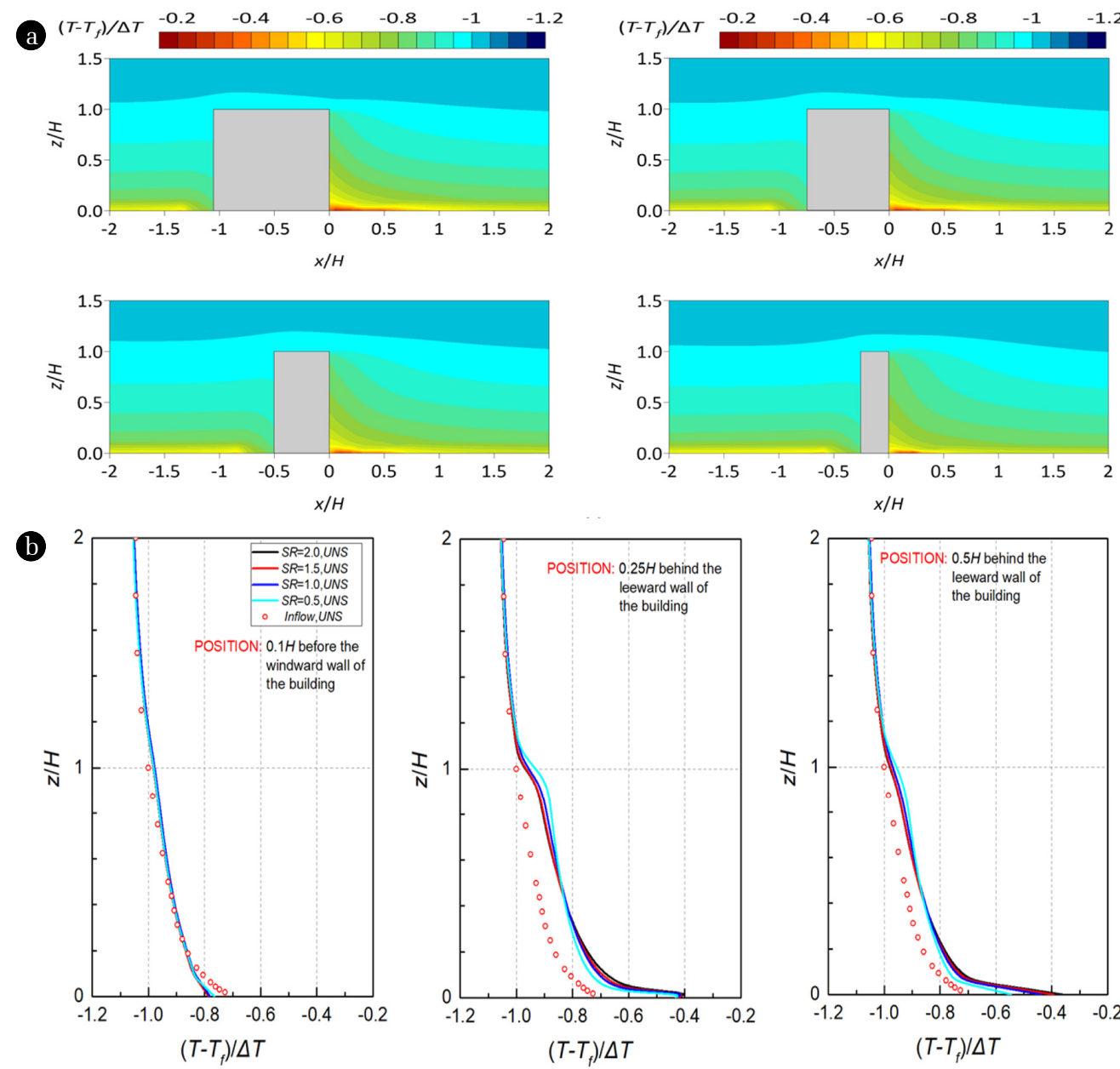

we strong downdraft on the windward side made the influence of the temperature stratification not obvious. On the leeward side of $0.25 H$ and $0.5 H$, the wind speed $\left|u / U_{H}\right|$ of the recirculation region increased with decreasing $S R$, and the extent of the increase was larger in unstable conditions.

\subsection{Normalized Temperature, $\left(\boldsymbol{T}-\boldsymbol{T}_{f}\right) / \Delta \boldsymbol{T}$}

Fig. 5 depicted the normalized temperature distribution of the vertical profile $(y / H=0)$ (under unstable temperature stratification). As shown in Fig. 5, in a case of unstable temperature stratification, when the floor temperature was higher than the air temperature, due to the strong rising flow along the leeward surface, the hot air was transported up and was mixed in the recirculation zones. As a result, the temperature in the recirculation zones was increased. Moreover, with the decrease in $S R$, the vortex became larger and more stable, as if the temperature gradient in the recirculation region was smaller. This indicated that the temperature distribution behind the building was largely influenced by air movement. As the air flow resumed, the temperature stratification was gradually restored.

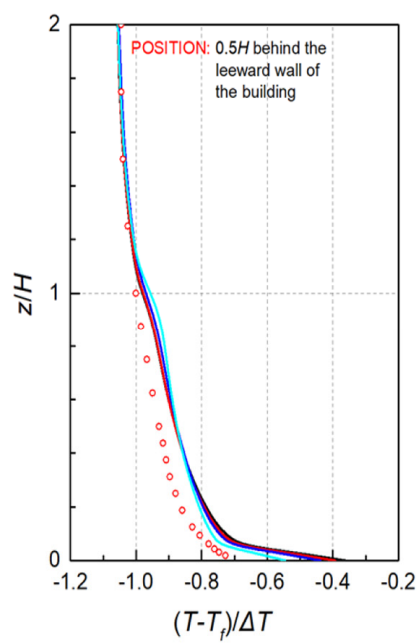

Fig. 5. (a) Normalized temperature distribution $\left(\left(T-T_{f}\right) / \Delta T\right)$ of vertical section $(y / H=0)$, and (b) of fixing points before $(0.1 H$ before the windward side) and behind the building $(0.25 \mathrm{H}$ and $0.5 \mathrm{H}$ behind the leeward side). 


\subsection{Normalized Turbulent Kinetic Energy, $k / U_{H}^{2}$}

Fig. 6(a) presented the distribution of the normalized turbulent kinetic energy $k / U_{H}^{2}$ in the vertical profile $(y / H=0)$. Fig. 6(b) showed the vertical distribution of $k / U_{H}^{2}$ at different positions in the vertical section $(y / H=0)$. In Fig. 6(a), under unstable temperature stratification conditions, $k / U_{H}^{2}$ appeared to be at a maximum near the windward angle of the building. However, under neutral conditions, the maximum $k / U_{H}^{2}$ occurred on the top of the building near the windward angle. The maximum value was obviously smaller than that under unstable temperature stratification conditions. It could be observed that $k / U_{H}^{2}$ became lager with increasing $R_{b}$, especially at the windward angle of the building. The reason for
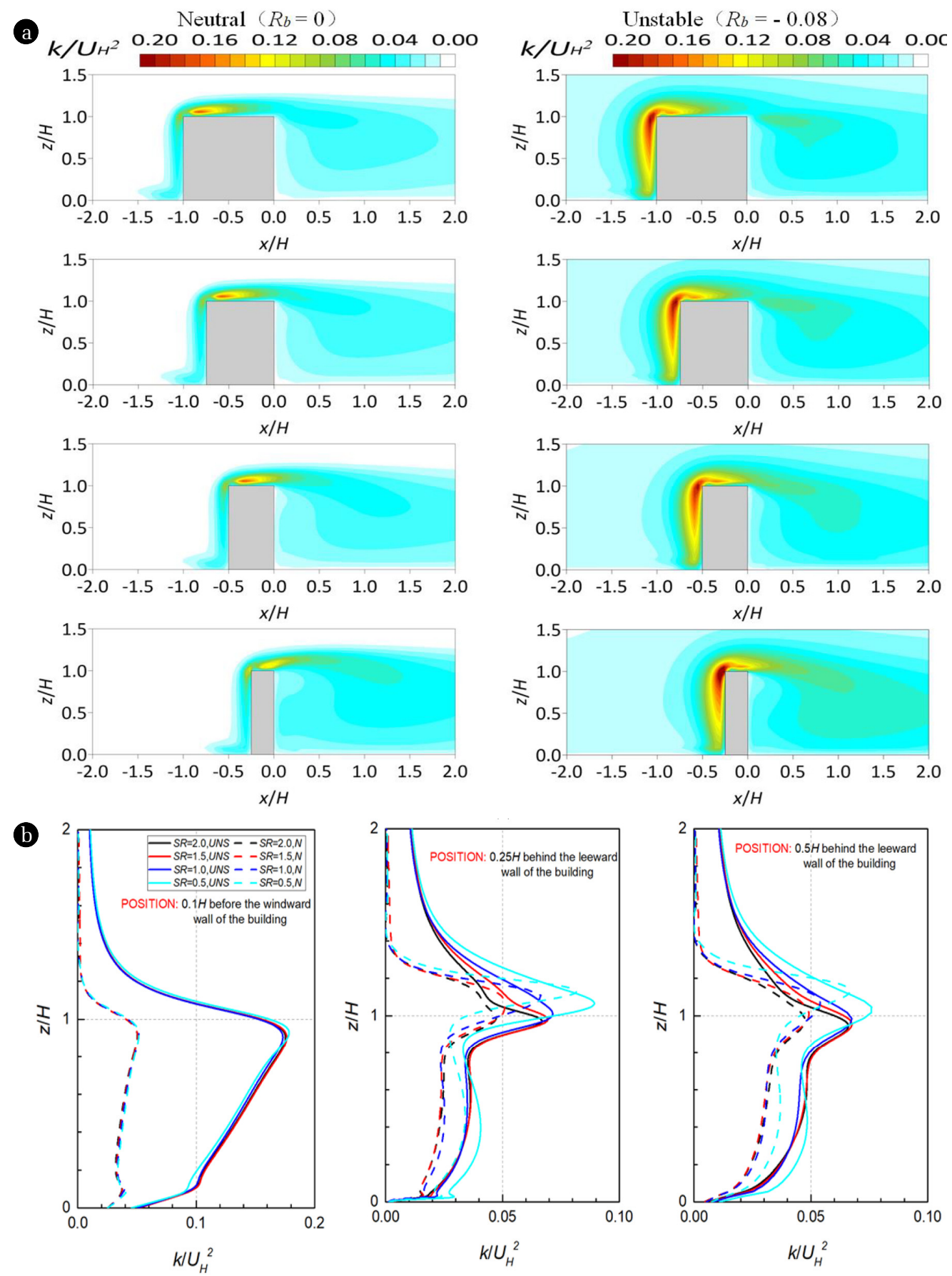

Fig. 6. Normalized turbulent kinetic energy distributions $\left(k / U_{H}^{2}\right)(\mathrm{a})$ of vertical section $(y / H=0)$, and (b) of fixing points before $(0.1$ $H$ before the windward side) and behind the building $(0.25 \mathrm{H}$ and $0.5 \mathrm{H}$ behind the leeward side). 
this might be due to the separation bubble created by the sharp edges of the building. The velocity difference between the high momentum flow and the stagnant flow on the roof of the building resulted in a high velocity gradient.

Fig. 6(b) revealed that at $0.1 \mathrm{H}$ before the windward side of the building, the effect of $S R$ on $k / U_{H}^{2}$ was negligible, but the effect of the stability was quite significant. However, both the temperature stratification conditions and $S R$ had obvious effects on $k / U_{H}^{2}$ behind the building. The peak values of $k / U_{H}^{2}$ increased with the decrease in $S R$ in both neutral and unstable temperature stratification at $0.25 \mathrm{H}$ or at $0.5 \mathrm{H}$ downwind of the building.

\subsection{Normalized Concentration Field, $C / C_{0}$}

Fig. 7(a) presented the normalized near-ground concentration of the $C / C_{0}$ distribution $(z / H=0.025)$. Overall, the pollutant plumes showed a similar distribution pattern in all simulations because the source of the pollution was located within the recirculation zone of the building. A higher concentration accumulated near the leeward of the building. However, some differences were still observed. For cases with large $S R$ values, high concentrations were observed throughout the wake area behind the building, especially in the region between the leeward wall and the source location. This was because the larger the $S R$ was, the smaller the vortex on the leeward side of the building, and the weaker the pollutant transport capacity, causing a large number of pollutants to accumulate on the leeward wall of the building after discharge. For cases with small $S R$ values, the more intense the vortex on the leeward side of the building, the more pollutants were transported to the side than downstream, while the pollutants behind the building were relatively less.

According to the concentration distribution diagram of the near-ground axis (Fig. 7(b)), the concentration decreased with the drop in $S R$. This characteristic was similar under both neutral and unstable conditions. In addition, with the decrease in $S R$, the width of the pollutant plume widened gradually. The reason for this was that with the decrease in $S R$, the reverse flow velocity increased, the transverse width of the recirculation zone increased, and the lateral wind speed near the ground of the building strengthened. This was consistent with the velocity distribution results. When the same $S R$ was present, the near-ground concentration of pollutants under unstable conditions was smaller than that under neutral conditions. This was because under the condition of unstable temperature stratification, the vertical movement was relatively strong, and the pollutants were transmitted to the top of the building with a strong updraft on the leeward side of the building.

The distribution of the concentration fluxes represented the essentials of concentration transportation. Scalar transport of concentration consisted of convective and turbulent diffusion effects, which were expressed by convection as the mean scalar fluxes and turbulent diffusion fluxes, respectively. The convective fluxes and the turbulent diffusion fluxes were expressed as $Q_{c}$ and $Q_{t}$, respectively. The convective fluxes $Q_{c}$ were calculated by the mean velocities and mean concentration, while the turbulent diffusion fluxes $Q_{t}$ were modeled by the gradient diffusion hypothesis. Fig. 7(c) demonstrated the lateral direction normalized convective fluxes
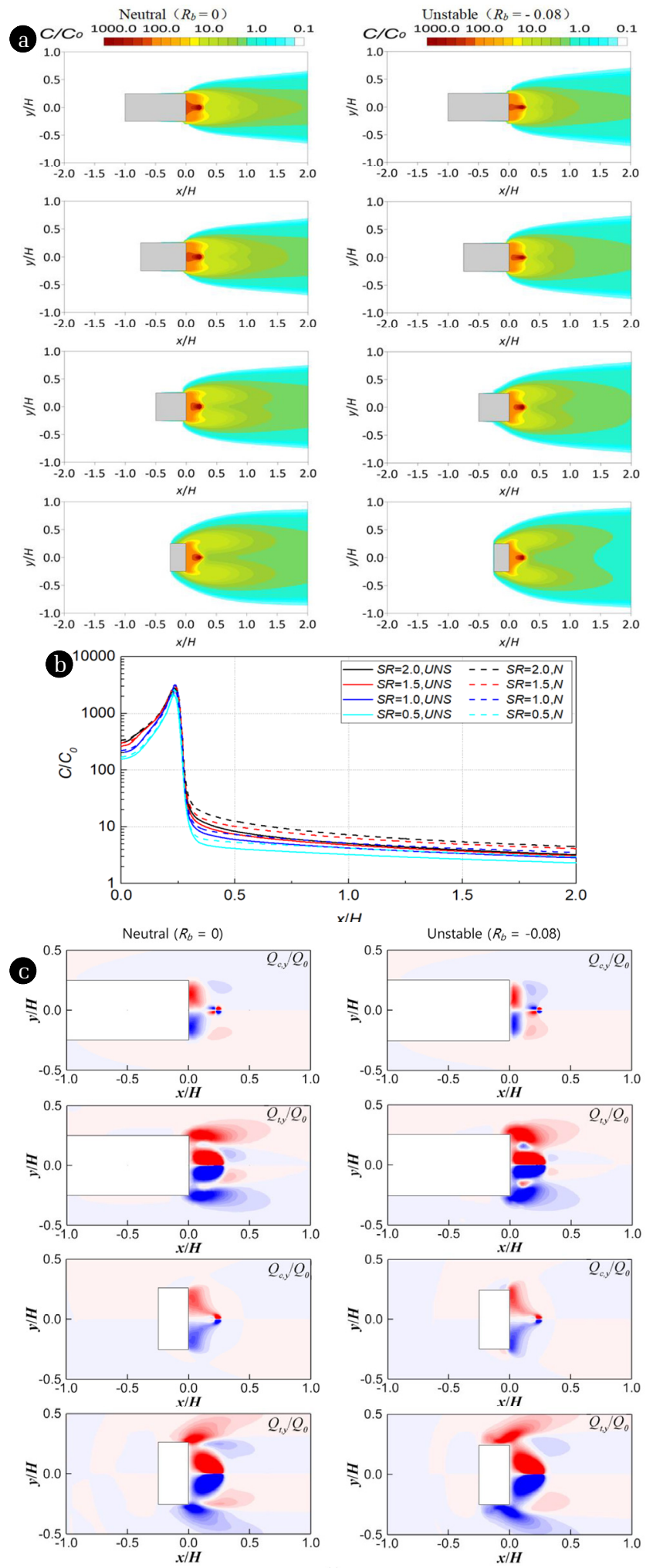

Fig. 7. Normalized concentration distribution (a) of near-surface $(z / H$ $=0.025)(b)$ of line $(y / H=0, z / H=0.025)$ and (c) lateral direction normalized convective fluxes $\left(Q_{c} / Q_{0}\right)$ and the turbulent diffusion fluxes $\left(Q_{t} / Q_{0}\right)$ distribution. 
$\left(Q_{c} / Q_{0}\right)$ and the turbulent diffusion fluxes $\left(Q_{c} / Q_{0}\right)$ distribution near the ground $(z / H=0.025)$ (a reference flux magnitude was used to make the convective and turbulent mass fluxes nondimensional. $Q_{0}=C_{0} U_{H}$ ). It was clear that both the convective flux and turbulent diffusion flux played important roles in lateral diffusion, and the latter even played a leading role. Under unstable conditions, the convection fluxes and turbulent fluxes of the side surface of the building were increased compared with those under neutral conditions. Although not fully demonstrated, the convective fluxes and turbulent fluxes increased as the $S R$ decreased. The convection fluxes were mainly related to the velocity field and the position and intensity of the vortex center. When $S R=2$, the vortex center was located upstream of the pollution source, while when $S R=0.5$, it was located downstream of the pollution source (see Fig. 4), so the distribution was different. However, turbulence stretched the plume in the lateral direction ( $y$-axis), and unstable conditions and a smaller $S R$ favored this stretching.

\section{Conclusions}

Based on the Reynolds-averaged Navier Stokes (RANS) method, the standard $k-\varepsilon$ turbulence model was used to simulate the effects of the side ratio ( $S R$ : building depth/building width, $S R=0.5$, $1.0,1.5$, and 2.0, respectively) of isolated buildings on the flow field and pollutant (ethylene) dispersion under unstable temperature stratification and neutral conditions. The main conclusions were as follows:

(i) The model validation results revealed that the velocity, temperature and concentration distribution calculated by CFD were basically consistent with the wind tunnel experiment results, which indicated that CFD could simulate unstable temperature stratification. For the case where the source of pollution was located downstream of the leeward side of the building, the SKE model showed the best agreement with the wind tunnel experiment results due to the best reproducibility of the velocity field in weak wind regions.

(ii) Temperature stratification and $S R$ had a great influence on the airflow, especially on the lateral wind direction and the downwind direction of the building. For a given temperature stratification condition, with the decrease in SR, the length of the backflow zone increased, and the lateral wind speed near the ground of the building was strengthened to some extent. For a given $S R$, the length of the recirculation zone was smaller under unstable temperature stratification conditions than under the neutral conditions.

(iii) In the case of unstable temperature stratification, when the floor temperature was higher than the air temperature, the strong rising flow along the leeward surface moved the hot air up and mixed it in the recirculation zones, causing the temperature in the recirculation zones to increase. Moreover, with the decrease in $S R$, the vortex became increasingly stable, and the temperature gradient in the recirculation region was smaller.

(iv) Temperature stratification and $S R$ had a strong influence on the peak value of the normalized turbulent kinetic energy $k / U_{H}^{2}$. For a given $S R$, the peak value of $k / U_{H}^{2}$ increased with increasing

$R_{b}$, especially in the windward angle of the building. The peak value of $k / U_{H}^{2}$ increased with the decline in $S R$ in both neutral and unstable temperature stratification conditions.

(v) Both convective fluxes and turbulent diffusion fluxes played important roles in lateral diffusion, and the latter even played a leading role. Turbulence stretched the plume in the lateral direction (y-axis), and unstable conditions and a smaller SR favored this stretching. The pollutant (ethylene) plumes showed a similar distribution pattern in all simulations under the circumstance that the source of pollution was located within the recirculation zone of the building. However, some differences were still observed. For a given $S R$, the near-ground concentration of pollutants under unstable conditions was lower than that under neutral conditions. For a given temperature stratification, the concentration decreased, but the width of the pollutant plume increased gradually with a decline in $S R$.

\section{Nomenclature}

C Gas concentration, ppmv

$C_{0}$

$C_{\text {gas }}$

$C / C_{0}$

$D$

$g$

$H$

$k$

$k / U_{H}^{2}$

$Q_{0}$

$Q_{c}$

$Q_{t}$

$Q_{c} / Q_{0}$

$Q_{t} / Q_{0}$

$Q_{V}$

$R_{b}$

$S c_{t}$

$S R$

$T$

$T_{H}$

$T_{f}$

$\mathrm{T}_{g a s}$

$\Delta T$

\section{$\left(T-T_{f}\right) / \Delta T$ Normalized temperature}

$u, v, w \quad$ Component of the velocity in the $x, y, z$ direction, $\mathrm{m} / \mathrm{s}$

$U_{H}$

$u^{*}$

$W$

$z$

$Z_{0}$

$\varepsilon$

$\kappa$

Reference gas concentration, $C_{0}=\frac{C_{g a s} \times Q_{V}}{U_{H} \times H^{2}} \mathrm{ppmv}$

Released tracer gas concentration, $C_{g a s}=5 \times 10^{4} \mathrm{ppmv}$

Normalized gas concentration

Depth of Building, mm

Gravitational acceleration, $\mathrm{m} / \mathrm{s}^{2}$

Building height, mm

Turbulent kinetic energy, $\mathrm{m}^{2} / \mathrm{s}^{2}$

Normalized turbulent kinetic energy

Reference flux magnitude, $Q_{0}=C_{0} U_{H}$

Convective fluxes

Turbulent diffusion fluxes

Normalized convective fluxes

Normalized turbulent diffusion fluxes

Released gas emission amount, $\mathrm{m}^{3} / \mathrm{s}$

Bulk Richardson number, $R_{b}=\frac{g \times H \times\left(T_{H}-T_{f}\right)}{T_{f} \times U_{H}{ }^{2}}$

The turbulent Schmidt number

Building depth/building width, $S R=D / W$

Temperature, $\mathrm{K}$

Air temperature at building height, $\mathrm{K}$

Surface temperature of wind tunnel floor, $\mathrm{K}$

Temperature of released tracer gas, $\mathrm{K}$

Absolute value of temperature difference,

$\Delta T=\left|T_{H}-T_{f}\right|, \mathrm{K}$

Scalar speed at height $H, \mathrm{~m} / \mathrm{s}$

Ground friction velocity, $\mathrm{m} / \mathrm{s}$

Building width, mm

Height, mm

Ground roughness, $\mathrm{m}$

Dissipation rate of turbulent kinetic energy, $\mathrm{m}^{2} / \mathrm{s}^{3}$

Von Karman constant, $\kappa=0.41$ 


\section{Author Contributions}

H.X.H. (Ph.D. student) conducted all the simulation, validation, analysis, date sorting, visualization and wrote the manuscript. G.L.Z. (Professor) supervised the numerical simulation process, and revised the manuscript.

\section{References}

1. Gu ZL, Zhang YW, Cheng Y, Lee SC. Effect of uneven building layout on air flow and pollutant dispersion in non-uniform street canyons. Build. Environ. 2011;46(12):2657-2665.

2. Karra S, Malki-Epshtein L, Neophytou MKA. Air flow and pollution in a real, heterogeneous urban street canyon: a field and laboratory study. Atmos. Environ. 2017;165:370-384.

3. Nosek Š, Fuka V, Kukačka L, Kluková Z, Jaňour Z. Street-canyon pollution with respect to urban-array complexity: The role of lateral and mean pollution fluxes. Build. Environ. 2018;138: 221-234.

4. Tan W, Li C, Wang K, Zhu G, Liu L. Geometric effect of buildings on the dispersion of carbon dioxide cloud in idealized urban street canyons. Process Saf. Environ. Prot. 2019;122:271-280.

5. Hertwig D, Gough HL, Grimmond S, Barlow JF, Kent CW, Lin WE. Wake characteristics of tall buildings in a realistic urban canopy. Bound-Layer Meteorol. 2019;172:239-270.

6. Reiminger N, Vazquez J, Blond N, Dufresne M, Wertel J. CFD evaluation of mean pollutant concentration variations in step-down street canyons. J. Wind Eng. Ind. Aerod. 2020;196: 104032.

7. Hang J, Li Y, Sandberg M, Buccolieri R, Di Sabatino S. The influence of building height variability on pollutant dispersion and pedestrian ventilation in idealized high-rise urban areas. Build. Environ. 2012;56:346-360.

8. Duan G, Ngan K. Sensitivity of turbulent flow around a 3-D building array to urban boundary-layer stability. J. Wind Eng. Ind. Aerod. 2019;193:103958.

9. Marucci D, Carpentieri M. Dispersion in an array of buildings in stable and convective atmospheric conditions. Atmos. Environ. 2020;222:117100.

10. Oke TR. Street design and urban canopy layer climate. Eng. Build. 1988;11:103-113.

11. Kanda I, Yamao Y. Passive scalar diffusion in and above urban-like roughness under weakly stable and unstable thermal stratification conditions. J. Wind Eng. Ind. Aerod. 2016;148: 18-33.

12. Zhang K, Chen G, Wang X, et al. Numerical evaluations of urban design technique to reduce vehicular personal intake fraction in deep street canyons. Sci. Total Environ. 2019;653:968-994.

13. Shu C, Wang L, Mortezazadeh M. Dimensional analysis of Reynolds independence and regional critical Reynolds numbers for urban aerodynamics. J. Wind Eng. Ind. Aerod. 2020;203: 104232.

14. Keshavarzian E, Jin R, Dong K, Kwok KCS, Zhang Y, Zhao M. Effect of pollutant source location on air pollutant dispersion around a high-rise building. Appl. Math. Model. 2020;81: 582-602.

15. Aristodemou E, Boganegra LM, Mottet L, et al. How tall buildings affect turbulent air flows and dispersion of pollution within a neighbourhood. Environ. Pollut. 2018;233:782-796.

16. Richter A, Ruck B, Mohr S, Kunz M. Interaction of severe convective gusts with a street canyon. Urban Clim. 2018;23:71-90.

17. Li WJ, He YP, Zhang YW, et al. LES simulation of flow field and pollutant dispersion in a street canyon under time-varying inflows with time varying-SIMPLE approach. Build Environ. 2019;157:185-196.

18. Michioka T, Takimoto H, Ono H, Sato A. Large-eddy simulation of the effects of wind-direction fluctuations on turbulent flow and gas dispersion within a cubical canopy. Bound-Layer Meteorol. 2019;173(2):243-262.

19. Wood CR, Lacser A, Barlow JF, et al. Turbulent flow at $190 \mathrm{~m}$ height above London during 2006-2008: A climatology and the applicability of similarity theory. Bound.-Lay. Meteorol. 2010;137(1):77-96.

20. Marucci D, Carpentieri M, Hayden P. On the simulation of thick non-neutral boundary layers for urban studies in a wind tunnel. Int. J. Heat Fluid Fl. 2018;72:37-51.

21. Uehara K, Murakami S, Oikawa S, Wakamatsu S. Wind tunnel experiments on how thermal stratification affects flow in and above urban street canyons. Atmos. Environ. 2000;34:1553-1562.

22. Santos JM, Reis NC, Goulart EV, Mavroidis I. Experimental investigation of averaging time effects on building influenced atmospheric dispersion under different meteorological stability conditions. Build. Environ. 2009;44(6):1295-1305.

23. Yassin MF. A wind tunnel study on the effect of thermal stability on flow and dispersion of rooftop stack emissions in the near wake of a building. Atmos. Environ. 2013;65:89-100.

24. Boppana VBL, Xie ZT, Castro IP. Thermal stratification effects on flow over a generic urban canopy. Bound-Lay. Meteorol. 2014;153(1):141-162.

25. Tomas JM, Pourquie MJBM, Jonker HJJ. Stable stratification effects on flow and pollutant dispersion in boundary layers entering a generic urban environment. Bound-Lay. Meteorol. 2016;159(2):221-239.

26. Farzad B, Payam G, Mohsen K, Mohammad J. Large eddy simulation of thermal stratification effect on convective and turbulent diffusion fluxes concerning gaseous pollutant dispersion around a high-rise model building. J. Build. Perform. Simu. 2019;12(1):97-116.

27. Sessa V, Xie Z, Herring S. Thermal stratification effects on turbulence and dispersion in internal and external boundary layers. Bound.-Lay. Meteorol. 2020;176(1):61-83.

28. Tominaga Y, Stathopoulos T. Numerical simulation of dispersion around an isolated cubic building: comparison of various types of k- $\varepsilon$ models. Atmos. Environ. 2009;43:3200-3210.

29. Tominaga Y, Stathopoulos T. Numerical simulation of dispersion around an isolated cubic building: model evaluation of RANS and LES. Build. Environ. 2010;45:2231-2239.

30. Tominaga, Y. Flow around a high-rise building using steady and unsteady RANS CFD: Effect of large-scale fluctuations on the velocity statistics. J. Wind Eng. Ind. Aerod. 2015;142:93-103. 31. Tominaga Y, Stathopoulos T. Steady and unsteady RANS simu- 
lations of pollutant dispersion around isolated cubical buildings: effect of large-scale fluctuations on the concentration field. J. Wind Eng. Ind. Aerod. 2017;165:23-33.

32. Gousseau P, Blocken B, van Heijst GJF. CFD simulation of pollutant dispersion around isolated buildings: on the role of convective and turbulent mass fluxes in the prediction accuracy. J. Hazard. Mater. 2011;194:422-434.

33. Du YX, Blocken B, Pirker S. A novel approach to simulate pollutant dispersion in the built environment: transport-based recurrence CFD. Build. Environ. 2020;170:106604.

34. Mavroidis I, Griffiths RF, Jones CD, et al. Experimental investigation of the residence of contaminants in the wake of an obstacle under different stability conditions. Atmos. Environ. 1999;33:939-949.

35. Mavroidis I, Griffiths RF, Hall DJ. Field and wind tunnel investigations of plume dispersion around single surface obstacles. Atmos. Environ. 2003;37:2903-2918.

36. Mavroidis I, Andronopoulos S, Bartzis JG. Computational simulation of the residence of air pollutants in the wake of a 3-dimensional cubical building. The effect of atmospheric stability. Atmos. Environ. 2012;63:189-202.

37. Yassin MF. A wind tunnel study on the effect of thermal stability on flow and dispersion of rooftop stack emissions in the near wake of a building. Atmos. Environ. 2013;65:89-100.

38. Tominaga Y, Stathopoulos T. Ten questions concerning modeling of near-field pollutant dispersion in the built environment. Build. Environ. 2016;105:390-402.
39. Chew LW, Glicksman LR, Norford LK. Buoyant flows in street canyons: comparison of RANS and LES at reduced and full scales. Build. Environ. 2018;146:77-87.

40. Jiang G; Yoshie R. Side ratio effects on flow and pollutant dispersion around an isolated high-rise building in a turbulent boundary layer. Build. Environ. 2020;180:107078.

41. Mittal H, Sharma A, Gairola A. A review on the study of urban wind at the pedestrian level around buildings. J. Build. Eng. 2018;18:154-63.

42. Zhang YW, Gu ZL, Yu CW. Impact Factors on Airflow and Pollutant Dispersion in Urban Street Canyons and Comprehensive Simulations: a Review. Curr. Pollut. Rep. 2020;6:425-439.

43. Yoshie R, Tanaka H, Shirasawa T, Kurita T, Kobayashi T. Technique for simultaneous measurement of fluctuating concentration, velocity and temperature in non-isothermal flow. J. Environ. Eng. (Transactions of AIJ) 2008;628:799-806.

44. Tominaga Y, Mochida A, Yoshie R, et al. AIJ guidelines for practical applications of CFD to pedestrian wind environment around buildings. J. Wind Eng. Ind. Aerod. 2008;96:1749-1761.

45. Yoshie R, Jiang GY, Shirasawa T, Chung J. CFD simulations of gas dispersion around high-rise building in non-isothermal boundary layer. J. Wind Eng. Ind. Aerod. 2011;99:279-288.

46. Architectural Institute of Japan. AIJ Benchmarks for Validation of CFD Simulations Applied to Pedestrian Wind Environment around Buildings. Tokyo: Architectural Institute of Japan; 2016. 